ARTICLE

\title{
Continuous bioactivity-dependent evolution of an antibiotic biosynthetic pathway
}

\author{
Chad W. Johnston', Ahmed H. Badran (1) ${ }^{2}$ \& James J. Collins (10) 1,2,3,4,5,6凶
}

Antibiotic biosynthetic gene clusters (BGCs) produce bioactive metabolites that impart a fitness advantage to their producer, providing a mechanism for natural selection. This selection drives antibiotic evolution and adapts BGCs for expression in different organisms, potentially providing clues to improve heterologous expression of antibiotics. Here, we use phage-assisted continuous evolution (PACE) to achieve bioactivity-dependent adaptation of the $B G C$ for the antibiotic bicyclomycin (BCM), facilitating improved production in a heterologous host. This proof-of-principle study demonstrates that features of natural bioactivity-dependent evolution can be engineered to access unforeseen routes of improving metabolic pathways and product yields.

\footnotetext{
${ }^{1}$ Institute for Medical Engineering and Science, Massachusetts Institute of Technology, 77 Massachusetts Ave, Cambridge, MA 02139, USA. ${ }^{2}$ Broad Institute of MIT and Harvard, 415 Main St, Cambridge, MA 02142, USA. ${ }^{3}$ Wyss Institute for Biologically Inspired Engineering, Harvard University, 3 Blackfan Circle, Boston, MA 02115, USA. ${ }^{4}$ Department of Biological Engineering, Massachusetts Institute of Technology, 77 Massachusetts Ave, Cambridge, MA 02139, USA. ${ }^{5}$ Synthetic Biology Center, Massachusetts Institute of Technology, 77 Massachusetts Ave, Cambridge, MA 02139, USA. ${ }^{6}$ Harvard-MIT Program in Health Sciences and Technology, 77 Massachusetts Ave, Cambridge, MA 02139, USA. ${ }^{凶}$ email: jimjc@mit.edu
} 
A ntibiotic BGCs are found in nearly all sequenced bacteria, where their genes, proteins, and chemical products continuously undergo natural selection, evolving their fitnessenhancing bioactivities ${ }^{1}$. This bioactivity-dependent selection pressure can favor the retention and adaptation of BGCs acquired through horizontal gene transfer (HGT) from related organisms ${ }^{2}$, phylogenetically divergent bacteria ${ }^{3}$, or even other kingdoms of life $^{4}$. The sampling of untargeted mutations during natural BGC evolution contrasts with genetic refactoring methods used in synthetic biology that rely on regulatory element exchange and codon optimization to adapt BGCs and improve metabolite yield $^{5}$. Bioactivity-dependent directed evolution presents a complementary approach to improve metabolite production.

In vivo directed evolution requires strategies for mutation, selection, and amplification of genes of interest (GOIs). In nature, these processes are coupled and form the basis for Darwinian evolution through natural selection. Continuous directed evolution approaches have found utility in mirroring these coupled cycles of diversification and selection ${ }^{6-8}$, with phage-assisted continuous evolution (PACE) ${ }^{9}$ being one of the most well-studied methods ${ }^{10-12}$. In PACE, engineered bacteriophage carrying an evolving GOI persist in mutagenic lagoons where their infectivity is dependent upon the bioactivity of the GOI product, enabling continuous cycles of diversified replication and selection. Here, we extend these capabilities to the continuous directed evolution of an antibiotic BGC and demonstrate PACE's ability to drive mechanism-agnostic optimization of a biosynthetic pathway.

To investigate whether PACE could improve antibiotic production, we haven chose to work with the antibacterial agent bicyclomycin $(\mathrm{BCM})^{3,13,14}$, which is a unique inhibitor of the bacterial transcription termination factor $\mathrm{Rho}^{15}$. BCM is a modified 2,5-diketopiperazine, biosynthesized by a seven-enzyme pathway that is initiated by a tRNA-dependent cyclodipeptide synthase to form a cyclo(L-Ile-L-Leu) core that is subsequently oxidized by five non-heme iron-dependent monooxygenases and one cytochrome P450 monooxygenase ${ }^{14}$. First discovered in Streptomyces ${ }^{16}$, BCM BGCs have been identified in Gramnegative bacteria 3,17 , where they appear to have been acquired and adapted following $\mathrm{HGT}^{3}$. In this work, we demonstrate that this same bioactivity-dependent selection pressure can be used to adapt this BGC and improve the yield of its antibiotic product in a heterologous host.

\section{Results}

Development of a genetic circuit to detect BCM bioactivity. Positive selection during PACE requires linking gene expression to an evolving bioactivity. We developed a synthetic gene circuit wherein BCM production would trigger expression of a reporter protein in a dose-dependent manner. Our sensor features a constitutive promoter driving GFP transcription, interrupted by a Rho-dependent $\lambda \mathrm{tR} 1$ terminator. BCM-dependent Rho inhibition derepresses reporter transcription, resulting in GFP production (Fig. 1a, right). Addition of a BCM standard to Escherichia coli cells carrying this reporter revealed a robust correlation between BCM concentration and GFP fluorescence, with nearly 1000-fold dynamic range and sensitivity to antibiotic levels far below the MIC of $\sim 25 \mu \mathrm{g} \mathrm{mL}^{-1}$ (Fig. 1b). Reporter sensitivity and signal-to-noise could be altered by changing promoter strength and plasmid or terminator copy number (Supplementary Figs. 1 and 2).

To validate that this circuit design could facilitate bioactivitydependent positive selection in vivo, we tested it in the presence of an inducible BCM operon taken from Pseudomonas aerugi$n o s a^{13}$ (Fig. 1a). Comparison of E. coli carrying the sensitive BCM reporter plasmid pREP.1 and either a wild-type or catalytically inactivated $^{18}$ (BcmA S46A) BCM expression plasmid
(EP; pBCM.1) revealed 18-fold higher fluorescence in cells carrying the functional operon (Fig. 1c). Consistent with prior enzymatic characterization ${ }^{3,13,14}, \mathrm{BCM}$ production was optimal at $\leq 30^{\circ} \mathrm{C}$ and ablated at temperatures above $33^{\circ} \mathrm{C}$ (Supplementary Fig. 3). To demonstrate bioactivity-dependent selection and improve our signal-to-noise before PACE, mutations were introduced into PBCM.1 by error-prone PCR and passage through mutagenic E. coli, and resultant clones were induced in the presence of the GFP reporter and sorted by fluorescenceactivated cell sorting (FACS). This approach identified mutagenized operons with a fivefold improvement in reporter signal relative to the wild-type operon (Supplementary Fig. 4), providing an optimized starting point for PACE.

Bioactivity-dependent PACE of BCM biosynthesis. The stringent BCM reporter plasmid pREP.2 (Supplementary Fig. 1) was modified to create an accessory plasmid (AP) for PACE, such that increasing concentrations of $\mathrm{BCM}$ result in increasing expression of the phage infectivity protein pIII (the product of geneIII; Fig. 2a). Selection phage (SP) bearing our FACS-optimized BCM operon $\left(\mathrm{SP}_{\mathrm{BCM}}\right)$ efficiently produced progeny from infected $E$. coli S2060 cells carrying our AP, while those bearing the BcmA S46A mutant did not (Supplementary Fig. 5). To accommodate the observed temperature sensitivity, PACE campaigns were carried out at $30^{\circ} \mathrm{C}$ using the chemical mutagen 1-methyl-3-nitro-1nitrosoguanidine (MNNG) for mutagenesis (Fig. 2b). To maximize the chances of discovering highly functional BCM operons, we first subjected $\mathrm{SP}_{\mathrm{BCM}}$ to a high concentration of MNNG $\left(1 \mathrm{mg} \mathrm{mL}^{-1}\right)$ to diversify our starting population, and observed a decline in phage titers consistent with a high mutagenic burden (Fig. 2c). This diversified population was subjected to $144 \mathrm{~h}$ of PACE-equivalent to $>800$ cycles of phage replication-wherein mutagenesis rate and selection stringency were modified by changes to MNNG concentration and lagoon flow rate, respectively. Phage samples were collected at 72 and $144 \mathrm{~h}$, and evolved operons were assessed using GFP reporter assays (Supplementary Figs. 6 and 7).

Sequence analysis of our evolved operons revealed a diversified pool of active BGCs (Fig. 2d), including a series of clones marked by a $\mathrm{BcmC}$ R40C mutation that demonstrated over tenfold higher reporter signal relative to our wild-type operon (Fig. 2e, f). This improved yield was further supported by liquid chromatography-mass spectrometry (LC/MS) analysis of BCM in culture extracts (Fig. 2g). From 72 sequenced operons, we observed 51 new mutations, including 30 silent or noncoding mutations and 21 missense mutations, many of which were also observed in sequenced BCM operons in other organisms (Supplementary Data 1, Supplementary Data 2). To assess how these mutations were impacting BCM production, individual mutant genes were cloned into a wild-type operon and induced in the presence of our reporter (Supplementary Fig. 8), revealing that most sequentially acquired mutations worked additively to improve production. To determine whether improved protein solubility was responsible for the observed increase in BCM production, we used wild-type and mutant versions of our enzymes in a split T7 RNA polymerase complementation assay ${ }^{19}$ (Supplementary Fig. 9). Final mutant versions of our $\mathrm{Bcm}$ enzymes generally did not possess greatly improved solubility-aside from BcmE L120I S2G-but several of the intermediate mutant enzymes did, which appears to have facilitated access to new sets of mutations that improved BCM production at the cost of soluble expression. LC/MS analysis of BCM metabolites in cultures expressing the wild-type or highyield evolved operon revealed considerable changes in the relative abundance of key intermediates (Supplementary Fig. 10), 
a

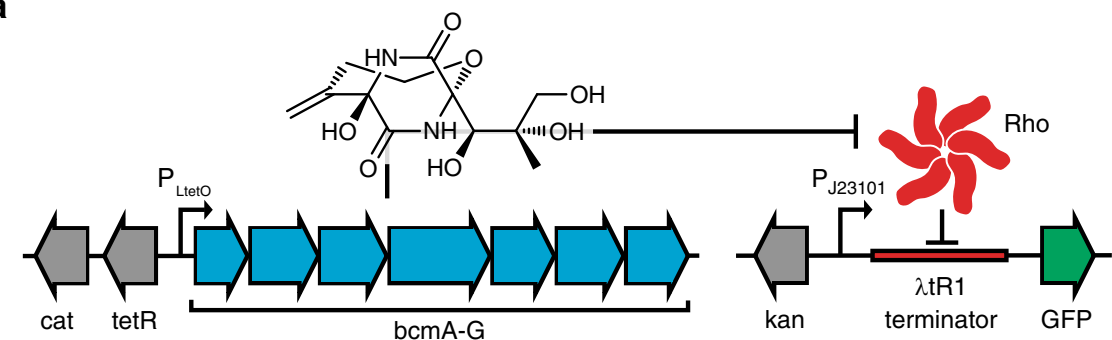

b

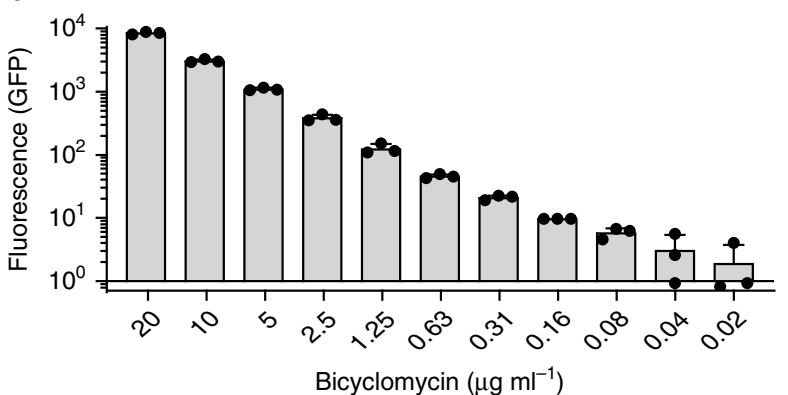

C

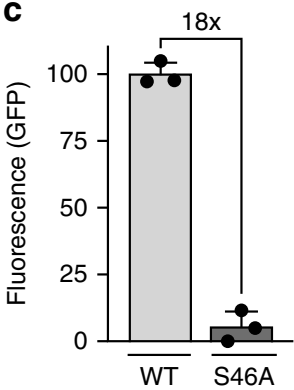

Fig. 1 A genetically encoded sensor for BCM bioactivity. a Schematic representation of plasmids for BCM production and BCM-dependent GFP expression, featuring a constitutive promoter interrupted by the Rho-dependent $\lambda t R 1$ terminator. BCM biosynthesis from a plasmid carrying an anhydrotetracycline (aTC) inducible promoter results in GFP production by alleviating Rho-dependent transcriptional termination of GFP on reporter plasmid pREP.1. b GFP reporter pREP.1 response across a wide range of $\mathrm{BCM}$ concentrations. c GFP production upon induction of the $P$. aeruginosa wild-type (WT) or inactivated (S46A) BCM operon. All data shown as mean \pm s.d.; $n=3$ biological replicates. Source data underlying $\mathbf{b}, \mathbf{c}$ are available as a Source Data file.

suggesting that adaptations in enzyme functionality compensate for the metabolic environment in $E$. coli. When these same operons are expressed in P. fluorescens SBW25-which is closely related to the organism from which this operon was sourcedwe did not observe improved BCM production (Supplementary Fig. 11), indicating again that PACE adapted this operon to improve $\mathrm{BCM}$ production in our heterologous host. While quantitative LC/MS analysis revealed a 20 -fold increase in yield between our evolved and wild-type operons in E. coli ( 0.6 and $0.03 \mu \mathrm{g} \mathrm{mL}^{-1}$, respectively), this effect was reversed in the previously described $P$. fluorescens BCM expression system (1.4 and $4.8 \mu \mathrm{g} \mathrm{mL}^{-1}$ respectively), where wild type yields closely matched those from the associated expression plasmid ${ }^{3}$ $\left(6 \mu \mathrm{g} \mathrm{mL}^{-1}\right.$, Supplementary Fig. 12).

Improving population fitness by tuning selection difficulty. PACE is unique among continuous evolution methods in that the replication of evolving material is uncoupled from host replication for genetic selections. This allows PACE to evolve potentially toxic biomolecules or phenotypes that could be harmful to E. coli, as naive cells are provided continuously, and their replication does not serve as the basis for selection. However, as our selection makes use of endogenous machinery, our capacity for selection is theoretically limited by the potency of the antibiotic and the abundance of its target. In order to dynamically modulate selection capacity and stringency during PACE-and limit potential toxicity downstream during production in $E$. coli-we tested a chemically inducible BCM efflux pump expression plasmid (bcmT; pPUMP). In cells carrying pPUMP, BcmT provided resistance to BCM (Supplementary Fig. 13a), and increasing expression resulted in lower reporter signal in response to BCM provided exogenously (Supplementary Fig. 13b) or produced inside the cell (Fig. 3a). To assess how active efflux would impact bioactivity-dependent evolution, we ran a $72 \mathrm{~h}$ PACE experiment, starting from our evolved phage pool. Reflecting the increased selection stringency imposed by active efflux of BCM during
PACE, we observed lower overall phage counts, along with retention and evolution of only our most productive operons from the evolved phage pool (Fig. 3, Supplementary Fig. 14). Plotting the populations of all recovered and assayed BGCs throughout our PACE experiments demonstrates that increasingly difficult selections can eliminate unproductive clones and more effectively drive a population-level shift toward increasingly active BGCs (Fig. 3d). As efflux pumps are associated with nearly all antibiotic BGCs, they could provide a universal mechanism to dynamically control selection difficulty during BGC evolution.

\section{Discussion}

Bioactivity-dependent evolution of antibiotic biosynthesis in PACE emulates natural selection by continuously introducing and sampling genetic diversity to promote fitness in the population. As each gene in a biosynthetic pathway contributes to producing a metabolite with a fitness-enhancing bioactivity, evolving the entire gene cluster simultaneously can provide access to unexpected mechanisms of improving yields that would not be identified by focusing on individual biosynthetic components ${ }^{20}$. Importantly, the mutations and adaptations accessed through bioactivity-dependent BGC evolution should be complementary to sequence optimizations featured in traditional genetic refactoring approaches ${ }^{5}$. Because PACE decouples the replication of the evolving GOI from the replication of hosts for selection, it can be used to evolve potentially toxic phenotypes-such as antibiotic production-that would limit other, less segregated systems, where selection depends on host viability and reproduction ${ }^{6-8}$.

Throughout this study, we observed that increasing the difficulty of a given genetic selection improved the overall activity of the evolving population at the cost of sequence diversity. As we had previously seen that epistatic mutations could provide access to improved properties-including protein solubility-it should be noted that strong selection pressure may not necessarily drive evolution toward a global fitness maxima. Conveniently, antibiotic BGCs possess conserved features that can be used 
a

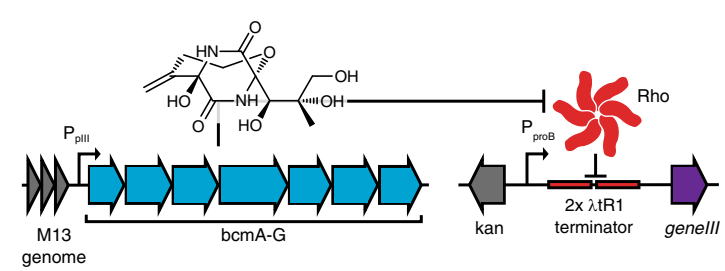

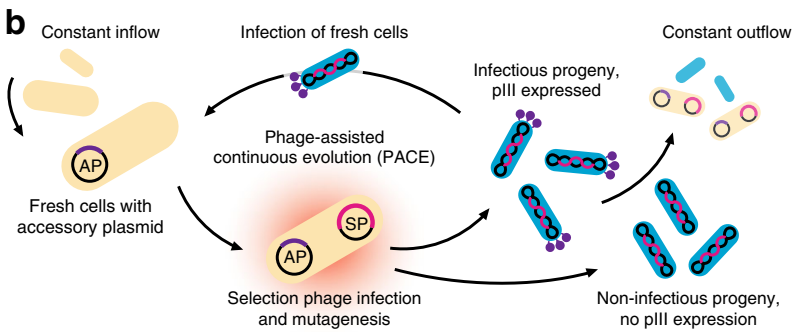

c

$\mathrm{AP}=2 \times \lambda \mathrm{tR} 1 \mathrm{proB}$

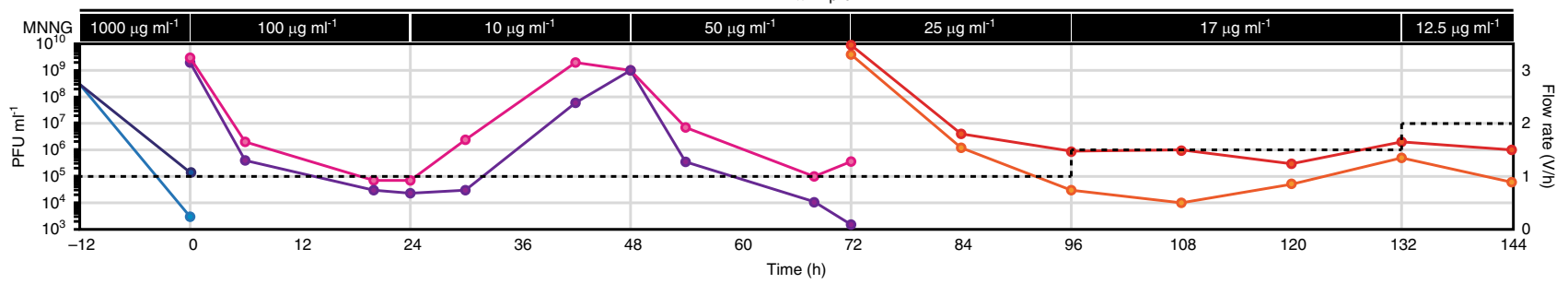

d

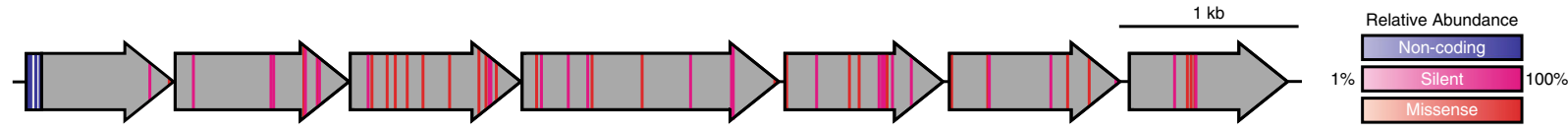

5' UTR bcmA bcmB

$\mathrm{bcmc}$

$\mathrm{bcmD}$

bcmE

$\mathrm{bcmF}$

$\mathrm{bcmG}$
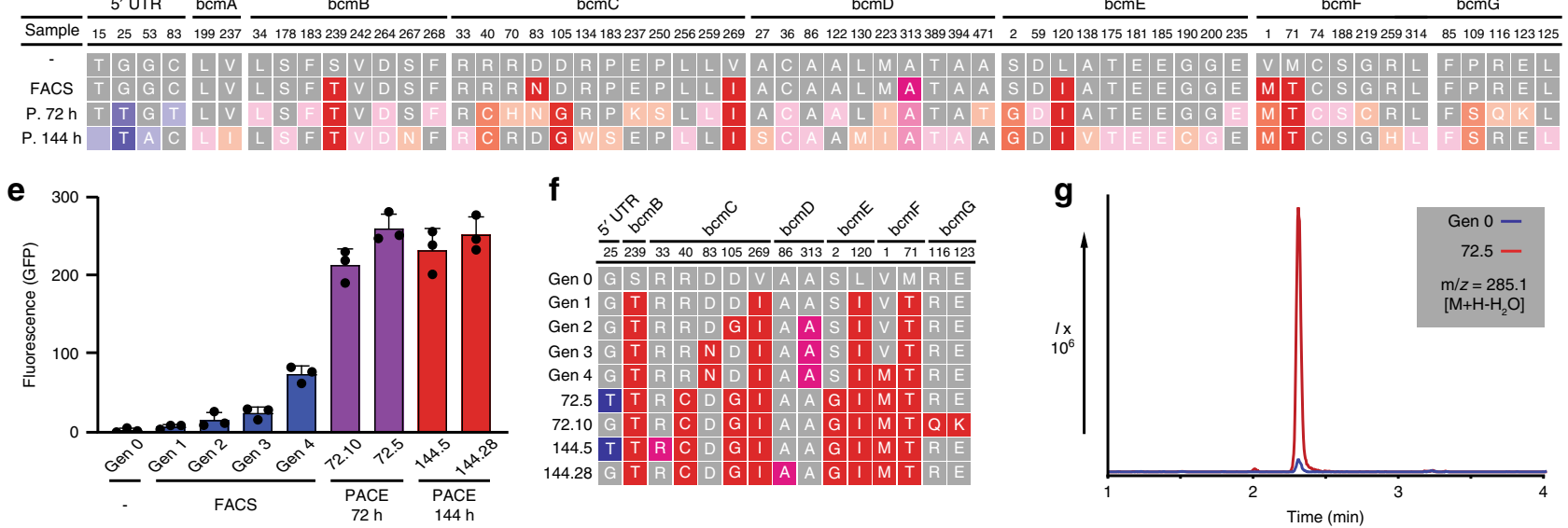

f

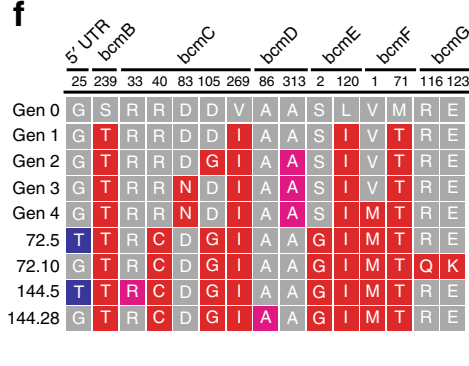

g

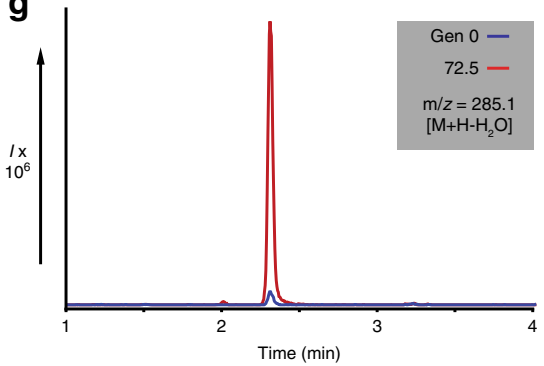

Fig. 2 Bioactivity-dependent continuous evolution of the P. aeruginosa BCM BGC. a Accessory plasmid and genetic circuit design for bioactivity-dependent expression of genellI. b Schematic representation of PACE. E. coli cells carrying an accessory plasmid (AP; purple) encoding genelll are provided continuously from a chemostat to fixed-volume lagoons inoculated with selection phage (SP; blue). Only functional SPs can propagate and persist within the lagoon under continuous dilution. Lagoons are supplemented with a chemical mutagen to facilitate mutagenesis at $30^{\circ} \mathrm{C}$. c $\mathrm{SP}_{\mathrm{BCM}}$ titers (plaque forming units; $\mathrm{PFU}$ ) over $144 \mathrm{~h}$ of PACE, reporting values for duplicate lagoons. Concentrations of the mutagen 1-methyl-3-nitro-1-nitrosoguanidine (MNNG) are indicated for each segment, along with lagoon flow rates (dashed lines). $\mathbf{d}$ Summary of mutations accumulated during BCM operon evolution. Below, relative abundance of each mutation is depicted. e Activity of wild-type and evolved BCM operons against the stringent reporter pREP.2 (Supplementary Fig. 1). Shown as mean \pm s.d.; $n=3$ biological replicates. $\mathbf{f}$ Genotypes of tested BCM variants. A legend for color coding can be found in $\mathbf{d} . \mathbf{g} \mathrm{LC} / \mathrm{MS}$ extracted ion chromatograms of the dominant bicyclomycin parent ion (an in-source decay water loss, $\mathrm{m} / \mathrm{z}=285.1\left[\mathrm{M}+\mathrm{H}-\mathrm{H}_{2} \mathrm{O}\right]$ ) from culture supernatants of the original $\mathrm{BCM}$ operon (Gen 0 ) and a PACE-evolved variant (72.5). Source data underlying c, e are available as a Source Data file.

to dynamically adjust selection pressure during continuous bioactivity-dependent evolution. Antibiotic resistance genes are endemic to $\mathrm{BGCs}^{21}$, where their expression is typically driven by a colocalized transcriptional regulator that is responsive to an associated antibiotic's chemical structure ${ }^{22}$ or to its specific mechanism of action ${ }^{23}$. Resistance enzymes that inactivate antibiotics or remove them from the cell can also be induced during directed evolution, such that their expression can be titrated to gradually adjust selection stringency. As we demonstrated with the induced expression of the BCM efflux pump $b c m T$, this additional pressure can be used to eliminate unproductive clones, limit potential cross-talk caused by antibiotic transmission between cells, and mitigate toxicity. While it had initially seemed likely that intracellular antibiotic accumulation during evolution was limiting our access to more productive sequences, analysis of clones recovered from PACE with active BCM efflux demonstrated that, in this case, toxicity was a minor limitation. Still, given that bioactivity-dependent continuous evolution relies on gene expression being linked to inhibition of an intracellular target, the upper limit on attainable antibiotic yields would be bound by the dynamic range of the genetic response to bioactivity, target affinity, antibiotic-associated toxicity, and the abundance and rates of antagonizing resistance enzymes.

Continuous directed evolution methods rely on genetic selections that are tailored to the current or desired function of the evolving GOI. For bioactivity-dependent continuous evolution, these selections instead rely on the activity of the small molecule produced by an evolving biosynthetic pathway. PACE mandates that the function of the GOI be linked to expression of the minorcoat protein PIII, a criterion that has been addressed with unique 
a

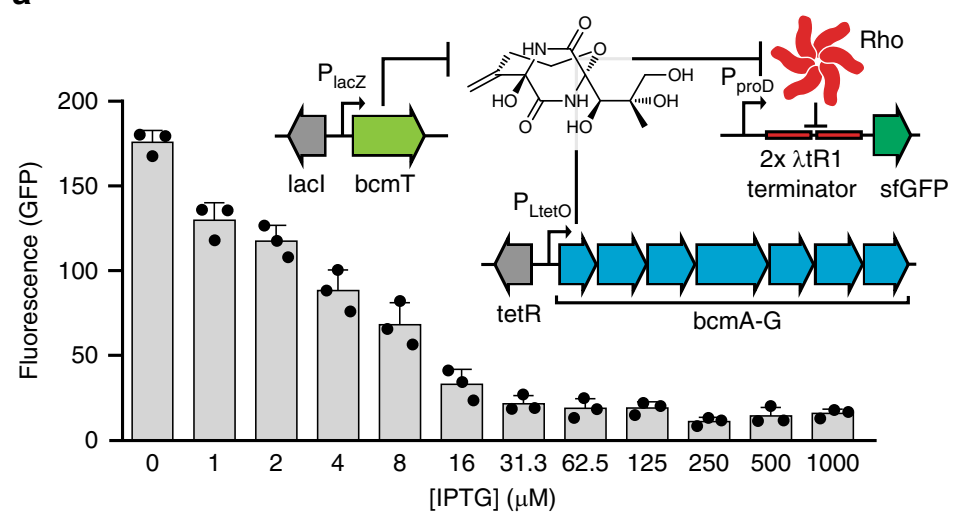

C

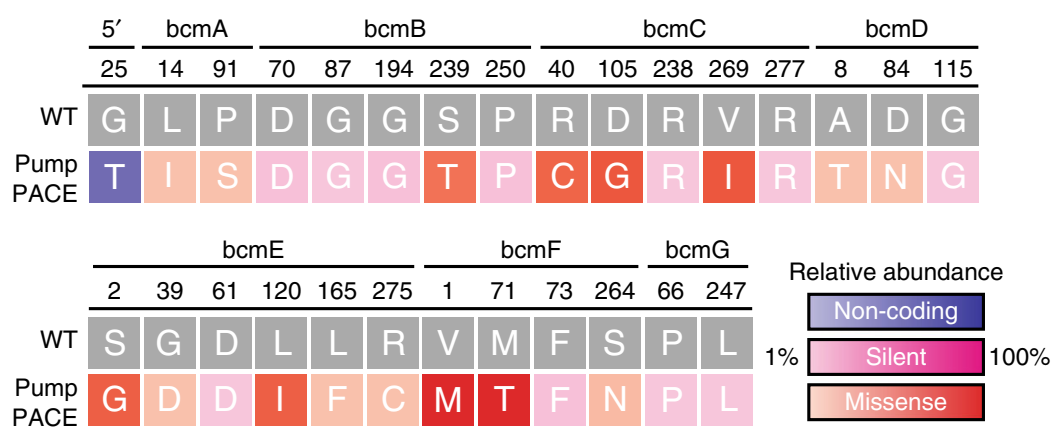

b

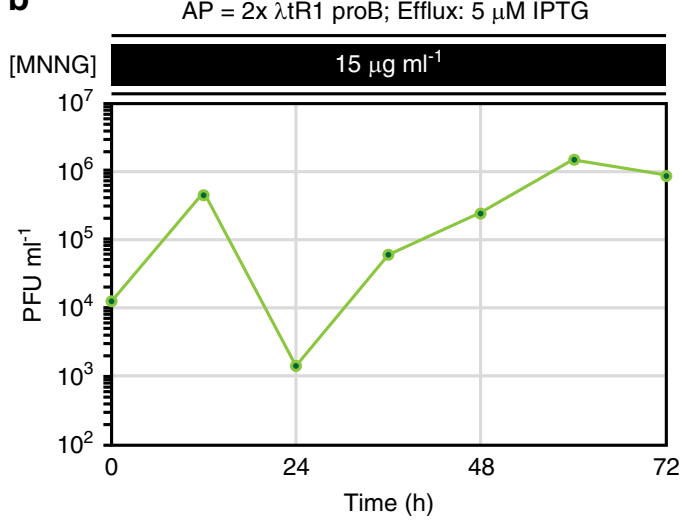

d

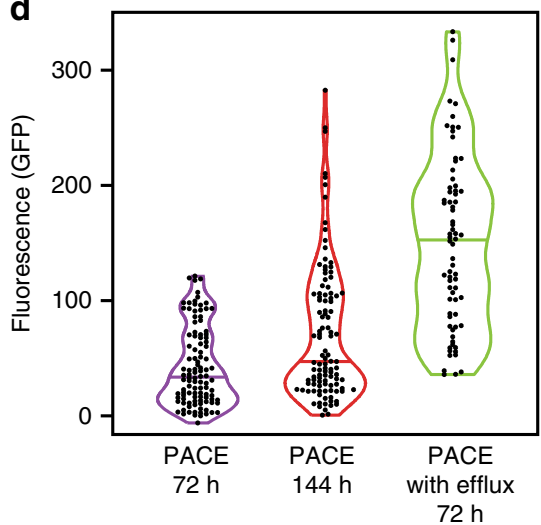

Fig. 3 BCM efflux during PACE yields a more productive population of BGCs. a Fluorescence (GFP) signal from BCM reporter plasmid pREP.2 in E. coli cells carrying the IPTG-inducible BCM efflux pump expression plasmid pPUMP and evolved BCM production plasmid pBCM.1 (recovered PACE clone 72.5). Results are absorbance normalized signal over uninduced controls, shown as mean \pm s.d.; $n=3$ biological replicates. $\mathbf{b} \mathrm{SP}_{\mathrm{BCM}}$ titers (plaque forming units, PFU) over an additional $72 \mathrm{~h}$ of PACE, using S2060 E. coli carrying accessory plasmid pAP.1 and the IPTG-inducible efflux pump expression plasmid pPUMP (induced with $5 \mu \mathrm{M} \mathrm{IPTG),} \mathrm{along} \mathrm{with} 15 \mu \mathrm{g} \mathrm{mL} \mathrm{L}^{-1} \mathrm{MNNG}$ for mutagenesis. Lagoon flow rate was maintained at $1 \mathrm{vol} \mathrm{h}^{-1}$. c Summary and relative abundance of mutations observed in BCM operons following PACE with active BCM efflux (Pump PACE) from 24 sequenced operons (Supplementary Fig. 14). d. Summary of assayed BCM operon populations recovered from PACE (Supplementary Figs. 6, 7, and 14). The median value is marked with a line in each violin plot. Source data underlying $\mathbf{a}, \mathbf{b}, \mathbf{d}$ are available as a Source Data file.

selection circuits in order to facilitate the evolution of nucleotidebinding $^{24}$ or nucleotide-modifying enzymes ${ }^{25}$, transcription ${ }^{9,11}$ and translation ${ }^{10}$ machinery, or protein-protein interactions ${ }^{12}$. In this proof-of-principle study, we demonstrated that the biosynthetic pathway for BCM could be evolved with PACE by leveraging BCM's unique mechanism of action. Although other antibiotics do not share the same this antibacterial mechanism, the selection tactic employed in this approachlinking antibiotic-mediated target inhibition to gene expressioncould be applied to many other antibiotic classes. Generalization of this platform thus relies on the development of new gene circuits that can select for inhibition of different biological targets $^{21,26}$, with the most readily accessible likely being those directly related to gene expression and the central dogma. Orthogonal systems ${ }^{27}$ for transcription and translation could be used to create gene circuits where enzyme inhibition does not impact cellular fitness, facilitating the evolution of antibiotics that affect RNA polymerases ${ }^{28}$, ribosomes ${ }^{29}$, and tRNA synthetases ${ }^{30}$, among others. Abstraction from biological function could be taken further by employing the established two-hybrid PACE system $^{12}$ to evolve BGCs for antibiotics that facilitate ${ }^{31}$ or disrupt protein-protein interactions ${ }^{32}$. Alternatively, transcriptional regulators of resistance genes can be known to detect the damage caused by specific antibiotics, and leveraging these proteins could provide a bioactivity-dependent means of evolving valuable drugs like carbapenems ${ }^{23}$. Ultimately, directed evolution schemes are primarily limited by the creativity and biological understanding behind the selection structure. As we demonstrated in this study, so long as gene expression can be tied to the activity of an evolving molecule or BGC, PACE could be a viable strategy to emulate natural bioactivity-dependent evolution and drive the improvement and adaptation of antibiotic biosynthetic pathways.

\section{Methods}

General methods. DNA cloning, BCM expression studies, and GFP assays were performed in NEB Turbo cells (New England Biolabs) grown in $2 \times$ LB media (BD Biosciences). All discrete infection assays, plaque assays, and PACE experiments were performed in E. coli S2060 cells ${ }^{11}$ grown in Davis Rich Media (DRM). For BCM production and LC/MS analysis, E. coli cells were grown in DRM, while $P$. fluorescens SBW25 cells were grown in BCM production media (BCMM) ${ }^{3}$. Plasmids and SPs were assembled with USER cloning, and amplified using Phusion U Hot Start DNA polymerase (Thermo Fisher Scientific). Antibiotics were used at the following concentrations: carbenicillin, $50 \mu \mathrm{g} \mathrm{mL}^{-1}$; tetracycline, $10 \mu \mathrm{g} \mathrm{mL}^{-1}$; chloramphenicol, $50 \mu \mathrm{g} \mathrm{mL}^{-1}$; kanamycin, $25 \mu \mathrm{g} \mathrm{mL}^{-1}$. DNA for Rho-dependent terminators and for assembling the P. aeruginosa BCM pathway was synthesized by Integrated DNA Technologies. A list of all primers used in this study is available in Supplementary Data 3, and a list of all plasmids used in this study is available in Supplementary Table 1.

Preparation and transformation of competent cells. For creating competent cells of NEB Turbo or S2060 E. coli, overnight cultures were inoculated into $50 \mathrm{~mL}$ $2 \times \mathrm{LB}$ media (BD Biosciences) and grown in an incubator set at $37^{\circ} \mathrm{C}$ and $290 \mathrm{rpm}$ to mid-log-phase (absorbance at $600 \mathrm{~nm}=0.5$ ). Cells were harvested by centrifugation at $4000 \mathrm{~g}$ at $4{ }^{\circ} \mathrm{C}$ for $10 \mathrm{~min}$. Cell pellets were resuspended in $1.25 \mathrm{~mL}$ of 
$2 \times$ TSS solution (LB media containing $10 \%$ PEG 3350, 5\% v/v DMSO, and $20 \mathrm{mM}$ $\mathrm{MgCl}_{2}$ ). Aliquots were frozen at $-80^{\circ} \mathrm{C}$ for later use.

For transformations, $50 \mu \mathrm{L}$ aliquots of cells suspended in TSS were thawed on ice and $7.5 \mu \mathrm{L}$ of $\mathrm{KCM}$ solution $\left(100 \mathrm{mM} \mathrm{KCl}, 30 \mathrm{mM} \mathrm{CaCl}_{2}\right.$, and $50 \mathrm{mM} \mathrm{MgCl}_{2}$ ) was added, along with the plasmid DNA or USER assembly reaction to be transformed. Cell-DNA mixtures were left on ice for $15 \mathrm{~min}$ before heat shock in a $42{ }^{\circ} \mathrm{C}$ water bath for $45 \mathrm{~s}$. Transformations were cooled on ice for $1 \mathrm{~min}$ before the addition of $750 \mu \mathrm{L}$ of $2 \times \mathrm{LB}$ and transfer to a shaking $37^{\circ} \mathrm{C}$ incubator for recovery over $45 \mathrm{~min}$. Following recovery, cells were centrifuged at $4000 \mathrm{~g}$, resuspended in a small volume of residual media, and plated on $2 \times$ LB plates containing appropriate antibiotics, growing out overnight at $37^{\circ} \mathrm{C}$.

To create electro-competent $P$. fluorescens SBW25 cells, single colonies were inoculated into $4 \mathrm{~mL}$ of $2 \times \mathrm{LB}$ media (BD Biosciences) in a $15 \mathrm{~mL}$ polypropylene culture tube and grown in an incubator set at $28^{\circ} \mathrm{C}$ and $300 \mathrm{rpm}$ overnight. On the following day, cells were harvested by centrifugation at $4000 \mathrm{~g}$ at $4^{\circ} \mathrm{C}$ for $10 \mathrm{~min}$. Cells were washed twice with ice-cold sterile $10 \%$ glycerol before being resuspended in $200 \mu \mathrm{L} 10 \%$ glycerol. Aliquots of $50 \mathrm{~mL}$ cell suspension mixed with DNA were electroporated with $2500 \mathrm{~V}$, resuspended in $1 \mathrm{~mL} 2 \times \mathrm{LB}$, and recovered at $28^{\circ} \mathrm{C}$ with shaking for $1 \mathrm{~h}$ before being plated on LB agar plates with appropriate antibiotics at $28^{\circ} \mathrm{C}$.

Taq mutagenesis. For error-prone PCR mutagenesis, the BCM operon was amplified with Taq DNA polymerase (New England Biolabs) in $96 \times 50 \mu \mathrm{L}$ reactions. PCR amplicons were isolated with a QIAquick PCR purification kit (Qiagen), treated with DpnI (New England Biolabs) to remove residual template DNA, and then gel extracted. This purified pool of mutated BCM operons was amplified again in $96 \times 50 \mu \mathrm{L}$ PCR reactions, using Phusion U polymerase and primers containing deoxyuridine to install USER-compatible termini for assembly. Amplicons were PCR purified, treated with DpnI, and gel extracted before USER assembly with an aTC-inducible plasmid backbone. $72 \times 10 \mu \mathrm{L}$ USER assembly reactions were pooled and transformed into fresh TSS-KCM competent NEB Turbo E. coli cells carrying our superfolder GFP BCM reporter plasmid (equivalent to $100 \mathrm{~mL}$ of culture with absorbance at $600 \mathrm{~nm}=0.5$ ). Following a $15 \mathrm{~min}$ incubation on ice, cells were heat shocked in a $42^{\circ} \mathrm{C}$ water bath for $45 \mathrm{~s}$. Transformations were cooled on ice for $1 \mathrm{~min}$ before being diluted into $500 \mathrm{~mL}$ of $2 \times \mathrm{LB}$, shaking in a $37^{\circ} \mathrm{C}$ incubator for $45 \mathrm{~min}$ to recover. Following this recovery, antibiotics were added for selection of reporter and BCM plasmids. Following overnight growth, this pooled transformation was diluted 1:100 into $100 \mathrm{~mL}$ of fresh $2 \times \mathrm{LB}$ with antibiotics and $100 \mathrm{ng} \mathrm{mL}^{-1}$ aTC to induce $\mathrm{BCM}$ production, and left growing for $12-16 \mathrm{~h}$ at $30^{\circ} \mathrm{C}$ and $290 \mathrm{rpm}$. After overnight outgrowth and induction, cells were diluted 1:1000 into $1 \times$ PBS and sorted using a BD FACSAriaII (BD Biosciences), gating forward and side scatter to enrich for single cells, then sorting based on superfolder GFP fluorescence intensity. Sorted cells suspended in PBS were plated on $2 \times$ LB agar with appropriate antibiotics and allowed to grow for $12-16 \mathrm{~h}$ at $37^{\circ} \mathrm{C}$. Three hundred eighty-four individual colonies of sorted cells were picked and resuspended into individual wells of a deep 96-well plate containing $2 \times \mathrm{LB}$ with antibiotics. Plates were grown at $37^{\circ} \mathrm{C}$ and $900 \mathrm{rpm}$ until cells reached early-mid $\log$ phase (absorbance at $600 \mathrm{~nm}=0.2$ ), after which individual wells were diluted into fresh media in four wells in a new plate, including duplicates with and without aTC. New plates were grown for $12-16 \mathrm{~h}$ at $30^{\circ} \mathrm{C}$ and $900 \mathrm{rpm}$ before cell density (absorbance at $600 \mathrm{~nm}$ ) and superfolder GFP fluorescence from the reporter was measured on a SpectraMax plate reader (Molecular Devices) to assess BCM production from mutated plasmids recovered following Taq mutagenesis.

XL-1 Red evolution. Fifty microliters aliquots of XL-1 Red E. coli cells (Agilent) were thawed on ice for $15 \mathrm{~min}$ before $1 \mu \mathrm{L}$ of a purified plasmid carrying an inducible copy of the BCM operon was added. Following 15 min incubation on ice, cells were heat shocked in a $42{ }^{\circ} \mathrm{C}$ water bath for $45 \mathrm{~s}$. Transformations were cooled on ice for $1 \mathrm{~min}$ before the addition of $750 \mu \mathrm{L}$ of $2 \times \mathrm{LB}$ and transfer to a shaking $37^{\circ} \mathrm{C}$ incubator for recovery over $45 \mathrm{~min}$. Following this recovery, transformations were plated on $2 \times \mathrm{LB}$ agar containing $50 \mu \mathrm{g} \mathrm{mL}^{-1}$ chloramphenicol and $10 \mu \mathrm{g} \mathrm{mL} \mathrm{m}^{-1}$ ketoconazole, incubating overnight at $37^{\circ} \mathrm{C}$. Colonies of transformants were washed off the plates and resuspended in $2 \times \mathrm{LB}$ containing matching antibiotics, diluted into a 96-well plate, and grown overnight at $37^{\circ} \mathrm{C}$. Every $24 \mathrm{~h}$ for 3 days, the plate was diluted 1:100 into new media and the remaining culture was used to isolate mutated BCM plasmids with a QIAprep Spin Miniprep kit (Qiagen). Following serial passaging and DNA prep, a pooled, mutated plasmid sample was transformed into competent NEB Turbo cells carrying a BCM-sensitive superfolder GFP reporter plasmid, and was allowed to recover in $100 \mathrm{~mL}$ of $2 \times \mathrm{LB}$ for $45 \mathrm{~min}$ before the addition of antibiotics, followed by outgrowth for $12-16 \mathrm{~h}$ at $37^{\circ} \mathrm{C}$ and $290 \mathrm{rpm}$. Following overnight outgrowth, transformed NEB Turbo cells carrying our BCM-sensitive reporter plasmid and our mutated BCM biosynthesis plasmid were diluted 1:100 into $100 \mathrm{~mL}$ fresh $2 \times \mathrm{LB}$ media with antibiotics and $100 \mathrm{ng} \mathrm{mL}^{-1}$ aTC to induce $\mathrm{BCM}$ production, and left growing for $12-16 \mathrm{~h}$ at $30^{\circ} \mathrm{C}$ and $290 \mathrm{rpm}$ After overnight outgrowth and induction, cells were diluted 1:1000 into $1 \times$ PBS and sorted using a BD FACSAriaII (BD Biosciences), gating forward and side scatter to enrich for single cells, then sorting based on superfolder GFP fluorescence intensity. Sorted cells suspended in PBS were plated on $2 \times$ LB agar with appropriate antibiotics and allowed to grow for $12-16 \mathrm{~h}$ at $37^{\circ} \mathrm{C}$. Three hundred eighty-four individual colonies of sorted cells were picked and resuspended into individual wells of a deep 96-well plate containing $2 \times \mathrm{LB}$ with antibiotics. Plates were grown at $37^{\circ} \mathrm{C}$ and $900 \mathrm{rpm}$ until cells reached early-mid $\log$ phase (absorbance at $600 \mathrm{~nm}=0.2$ ), after which individual wells were diluted into fresh media in four wells in a new plate, including duplicates with and without aTC. New plates were grown for $12-16 \mathrm{~h}$ at $30^{\circ} \mathrm{C}$ and $900 \mathrm{rpm}$ before cell density (absorbance at $600 \mathrm{~nm}$ ) and superfolder GFP fluorescence from the reporter were measured on a SpectraMax plate reader (Molecular Devices) to assess BCM production from mutated plasmids recovered from XL-1 Red transformants.

GFP reporter assays. Single colonies of NEB Turbo $E$. coli cells carrying a reporter plasmid and potentially also a plasmid containing an inducible BCM operon were used to create glycerol stocks. For assays, $2 \times$ LB containing appropriate antibiotics was inoculated with an appropriate stock, sampled with a sterile wooden stick. Cultures were grown overnight at $37^{\circ} \mathrm{C}$ and $250 \mathrm{rpm}$ to recover. On the following day, fresh media was inoculated 1:1000 with the overnight cultures grown in similar conditions to absorbance at $600 \mathrm{~nm}=0.6-0.8$ before being diluted $1: 100$ into $300 \mu \mathrm{L}$ of fresh media in a deep 96-well plate for assays. For reporter doseresponse assays, $2 \times \mathrm{LB}$ with antibiotics was serially diluted with $\mathrm{BCM}$ (dissolved in methanol; Santa Cruz Biotechnology) and warmed to $30^{\circ} \mathrm{C}$ prior to the addition of cells. For assays involving the induction of the $\mathrm{BCM}$ operon, $2 \times \mathrm{LB}$ with antibiotics and with or without $100 \mathrm{ng} \mathrm{mL}^{-1}$ aTC was added to a deep 96-well plate and warmed to the appropriate temperature $\left(30^{\circ} \mathrm{C}\right.$ in all cases beyond temperature dependence assays) prior to the addition of cells. Following inoculation, the assay plate was sealed with a breathable membrane and shaken at $900 \mathrm{rpm}$ for $12 \mathrm{~h}$. After incubation with shaking, assay plates were sampled, measuring fluorescence, and absorbance of the cultures on a SpectraMax plate reader (Molecular Devices). For assays involving the BCM efflux pump ( $b c m T)$, IPTG for induction of pump expression was added simultaneously with aTC and/or BCM.

Luciferase assay. S2060 cells were transformed with the pTW004(s) and pTW006 plasmids of interest, carrying the T7 RNA polymerase $\mathrm{N}$-terminal fusions of $\mathrm{Bcm}$ proteins, and the T7 C-terminal fragment and luciferase reporter, respectively ${ }^{19}$. Single colonies were grown overnight in DRM media supplemented with maintenance antibiotics, and were then diluted 1000-fold into DRM media with maintenance antibiotics in a deep 96 -well plate (VWR). The plate was sealed with a porous sealing film and grown at $37^{\circ} \mathrm{C}$ with shaking at $900 \mathrm{rpm}$ for $4 \mathrm{~h}$, whereupon the culture reached OD600 0.4-0.6. Cells were induced with $100 \mathrm{ng} \mathrm{mL}^{-1}$ aTC and $5 \mu \mathrm{M}$ arabinose before incubation for an additional $1 \mathrm{~h}$ at $37^{\circ} \mathrm{C}$ with shaking at $900 \mathrm{rpm}$, before $150 \mu \mathrm{L}$ of cells were transferred to a 96-well black-walled clearbottomed plate to measure absorbance at $600 \mathrm{~nm}$ and luminescence using a Tecan Spark microplate reader. OD600-normalized luminescence values were obtained by dividing raw luminescence by background-subtracted $600 \mathrm{~nm}$ absorbance. The background value was set to the $600 \mathrm{~nm}$ absorbance of wells containing DRM only.

Liquid chromatography and mass spectrometry. Mass spectrometry was performed using a Thermo QExactive mass spectrometer (ThermoFisher Scientific, USA) with a HESI II probe, operated in positive mode. Liquid chromatography was performed with a coupled Thermo Dionex UltiMate 3000 HPLC system (ThermoFisher Scientific, USA), using a Luna Omega $1.6 \mu \mathrm{m}$ Polar C18 column $(50 \times$ $2.1 \mathrm{~mm}$, Phenomenex) maintained at $25^{\circ} \mathrm{C}$. Mobile phases were water with $0.1 \%$ formic acid or methanol with $0.1 \%$ formic acid. For separations, organic solvent was increased from 0 to $35 \%$ over $6 \mathrm{~min}$, followed by a wash at $100 \%$ starting at $6 \mathrm{~min} 6 \mathrm{~s}$. BCM fragmentation and retention time were confirmed via analytical standard (Santa Cruz Biotechnology) prior to analysis. High-resolution mass spectrometry data were collected with full scan mode and with parallel reaction monitoring (PRM) for quantification. PRM targeting of BCM was accomplished by filtering for the associated ion $\left(\mathrm{m} / \mathrm{z}=285.1\left[\mathrm{M}+\mathrm{H}-\mathrm{H}_{2} \mathrm{O}\right]\right)$ and subsequent diagnostic MS/MS detection of diagnostic fragment ions. Intensities and peak areas of BCM were measured with MZmine2 software ${ }^{33}$ and concentrations were calculated by comparison with a standard curve.

For analysis of BCM production in E. coli, NEB Turbo cells were transformed with expression plasmid pBCM.1 or expression and efflux plasmid pBCM.2 bearing either the original BCM sequence or an evolved gene cluster recovered from PACE. Single colonies were grown overnight at $37^{\circ} \mathrm{C}$ and $300 \mathrm{rpm}$ in $4 \mathrm{~mL}$ DRM with appropriate antibiotics. Following overnight growth, a second set of $4 \mathrm{~mL}$ cultures was inoculated 1:100 and grown to absorbance at $600 \mathrm{~nm}=0.6-0.8$, then diluted 1:100 into $4 \mathrm{~mL}$ DRM with antibiotics and $100 \mathrm{ng} \mathrm{mL}^{-1}$ aTC and grown at $28^{\circ} \mathrm{C}$ and $300 \mathrm{rpm}$ for $16 \mathrm{~h}$. Cultures were harvested by centrifugation at $3200 \mathrm{~g}$ for $15 \mathrm{~min}$ at $4{ }^{\circ} \mathrm{C}$ using an Eppendorf 5804R centrifuge. Supernatants were sterilized by syringe filtration $(0.2-\mu \mathrm{m}$ filter) and cell pellets were resuspended in methanol, followed by a second centrifugation.

For analysis of BCM production in P. fluorescens, we followed the protocol outlined by Vior et al. ${ }^{3}$. Briefly, cells were transformed with Pseudomonas protein expression vector $\mathrm{pJH}$, carrying a wild-type or PACE-evolved BCM operon, along with the BCM efflux pump bcmT. Single colonies of the resulting transformations were used to inoculate $4 \mathrm{~mL}$ cultures of BCMM in $15 \mathrm{~mL}$ polypropylene culture tubes, growing overnight at $28^{\circ} \mathrm{C}$ and $300 \mathrm{rpm}$ Following overnight growth, a $400 \mu \mathrm{L}$ aliquot was diluted into $10 \mathrm{~mL}$ fresh BCMM in a $50 \mathrm{~mL}$ Falcon tube and covered with a foam bung. Production cultures were grown for $16 \mathrm{~h}$ at $28^{\circ} \mathrm{C}$ and 
$300 \mathrm{rpm}$ Cultures were harvested by centrifugation at $3200 \mathrm{~g}$ for $15 \mathrm{~min}$ at $4{ }^{\circ} \mathrm{C}$ using an Eppendorf 5804R centrifuge. Supernatants were sterilized by syringe filtration $(0.2-\mu \mathrm{m}$ filter $)$

Plaque assays. Single colonies of S2060 E. coli cells carrying the activityindependent persmissive plasmid pJC175 $\mathrm{e}^{11}$ were grown overnight in DRM media supplemented with antibiotics, before being diluted 1:1000 into fresh DRM media with antibiotics and grown at $37^{\circ} \mathrm{C}$ and $250 \mathrm{rpm}$ to absorbance at $600 \mathrm{~nm}=$ $0.6-0.8$ before use. Phage samples were serially diluted 1:100 four times in DRM media. $100 \mu \mathrm{L}$ of cells were added to $10 \mu \mathrm{L}$ of each phage dilution and allowed to sit for $\sim 1$ min before addition of $1 \mathrm{~mL}$ of warm liquid top agar $(2 \times \mathrm{YT}$ media $+0.4 \%$ agar) supplemented with $2 \%$ Bluo-gal (dissolved in DMF; Sigma) to a final concentration of $40 \mu \mathrm{g} \mathrm{mL}^{-1}$ was added and mixed by pipetting up and down once. The resuspended cells and phage dilution were then pipetted onto a quartered Petri dish already containing $2 \mathrm{~mL}$ of solidified bottom agar in each quadrant $(2 \times \mathrm{YT}$ media $+1.5 \%$ agar, no antibiotics). Plates were incubated at $37^{\circ} \mathrm{C}$ overnight after the top agar was observed to have solidified.

Phage propagation assays. Single colonies of S2060 cells carrying an AP of interest were prepared similarly to those used in activity-independent plaque assays. Following dilution into fresh media and growth at $37^{\circ} \mathrm{C}$ and $250 \mathrm{rpm}$ to absorbance at $600 \mathrm{~nm}=0.6-0.8,4 \mathrm{~mL}$ cultures of cells were divided into two new cultures and prepared SP were added to a final titer of $1 \times 10^{5} \mathrm{pfu} \mathrm{mL}^{-1}$. Cultures were moved to a $30^{\circ} \mathrm{C}$ shaker set to $250 \mathrm{rpm}$ and left for phage propagation. Phage counts were assessed after 2,4 , and $6 \mathrm{~h}$, collecting supernatants containing phage by transferring $100 \mu \mathrm{L}$ of culture to a $1.7 \mathrm{~mL}$ Eppendorf tube, centrifuging at $2000 \mathrm{~g}$ for $5 \mathrm{~min}$. Supernatants were filtered using sterile polyethersulfone $0.2-\mu \mathrm{m}$ centrifuge filters (mdi Membrane Technologies), centrifuging again at $2000 \mathrm{~g}$ for $5 \mathrm{~min}$. Samples were stored at $4{ }^{\circ} \mathrm{C}$ for up to 1 week before use.

Phage-assisted continuous evolution. The apparatus for PACE-including tubing, pumps, chemostats, and lagoons-was used as previously described ${ }^{9,11,12}$ unless noted otherwise. S2060 cells were transformed with the AP pAP.1 and plated on $2 \times \mathrm{LB}$ agar containing appropriate antibiotics, incubating overnight at $37^{\circ} \mathrm{C}$. A single colony of S2060 with the transformed AP was grown in $4 \mathrm{~mL}$ DRM with antibiotics at $37^{\circ} \mathrm{C}$ and $250 \mathrm{rpm}$ until absorbance at $600 \mathrm{~nm}=0.6-0.8$, when it was added to a $100 \mathrm{~mL}$ chemostat containing fresh media. The chemostat culture was grown until absorbance at $600 \mathrm{~nm}=0.6-1.0$, after which pumps were started to supply fresh media at a rate of $1-1.5 \mathrm{vol} \mathrm{h}^{-1}$ to maintain cell density. Lagoons were filled with DRM and constantly diluted with culture from the chemostat following initiation of the pumps. Lagoons were allowed to equilibrate for $\sim 8 \mathrm{~h}$ prior to the addition of phage. Unless stated otherwise, lagoons were maintained at a volume of $20 \mathrm{~mL}$ and fed at a flow rate of $1 \mathrm{vol} \mathrm{h}^{-1}$ from the chemostat. Any changes in dilution rate during PACE experiments were exclusively implemented through alterations to pumping rate, rather than adjusting lagoon volume. For PACE experiments involving the inducible $\mathrm{BCM}$ efflux pump plasmid pPUMP, chemostat DRM was modified with carbenicillin for plasmid maintenance and $5 \mu \mathrm{M}$ IPTG for constitutive induction.

Following equilibration of lagoon and chemostat cultures, lagoons were seeded

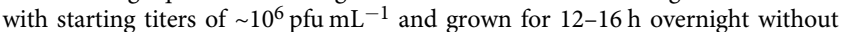
mutagenesis to reach high titers for the initiation of mutagenic PACE the following morning. After overnight culturing, lagoons were sampled prior to the addition of mutagen ( $t=0 \mathrm{~h}$ ) by removing $3 \mathrm{~mL}$ of culture via sterile syringe. For mutagenesis, MNNG (TCI America) was dissolved in DMSO for direct infusion and dilution into lagoons, pumped from $50 \mathrm{~mL}$ syringes through Versilon SE-200 chemical transfer tubing (Saint-Gobain) using a syringe pump (New Era Pump Systems) at a flow rate between 0.1 and $1.0 \mathrm{~mL} \mathrm{~h}^{-1}$. Following the initiation of mutagen addition, lagoons were sampled at regular intervals (roughly every $12 \mathrm{~h}$ ).

Samples taken from lagoons were processed immediately to isolate phage particles and dsDNA. One milliliter of culture sample was transferred to a $1.7 \mathrm{~mL}$ Eppendorf tube, centrifuging at $2000 \mathrm{~g}$ for $5 \mathrm{~min}$. Supernatants were filtered using sterile polyethersulfone $0.2-\mu \mathrm{m}$ centrifuge filters (mdi Membrane Technologies), centrifuging again at $2000 \mathrm{~g}$ for $5 \mathrm{~min}$. Samples were stored at $4{ }^{\circ} \mathrm{C}$ for up to 1 week before use. The remaining culture sample was centrifuged in the original $1.7 \mathrm{~mL}$ Eppendorf tube at $20,000 \mathrm{~g}$ for $1 \mathrm{~min}$ to collect the cell pellet for plasmid isolation. Genotype assessment and monitoring of the integrity of the BCM operon was performed regularly by PCR off of isolated phage dsDNA using primers BCM_CWI_USER_F (5'-AGGGGCCCAAG ${ }_{\mathrm{d}}$ UTCACTTAAAAAGGAG) and BCM_CWJ_USER_R (5'-ACTCTAGATCAATA $d$ UA-GACCCTGGGTATTCTC) and a standard thermocycler program $\left(98^{\circ} \mathrm{C} 1 \mathrm{~min}\right.$, followed by 30 cycles of $98^{\circ} \mathrm{C}$ for $10 \mathrm{~s}, 60^{\circ} \mathrm{C}$ for $30 \mathrm{~s}, 72{ }^{\circ} \mathrm{C}$ for $3 \mathrm{~min} 30 \mathrm{~s}$, followed by $72{ }^{\circ} \mathrm{C}$ for $10 \mathrm{~min}$ ). PCR products were assessed by $0.8 \%$ agarose gel electrophoresis. For operon recovery, the resulting PCR product was gel excised and cloned into an inducible plasmid backbone. Assembled BCM EPs were transformed into NEB Turbo cells carrying a fluorescent reporter and plated on $2 \times \mathrm{LB}$ agar containing appropriate antibiotic and $100 \mathrm{ng} \mathrm{mL}^{-1}$ aTC, growing at $30^{\circ} \mathrm{C}$ for $48-96 \mathrm{~h}$. GFP expression from individual colonies was monitored via inspection on a SafeImager 2.0 UV imager (Invitrogen). Colonies that expressed GFP were isolated, retested in liquid culture, and grown for plasmid isolation and Sanger sequencing (Quintarabio) to determine genotype.

Phage titers were determined by plaquing filtered culture supernatant samples on S2060 cells carrying the activity-independent permissive plasmid $\mathrm{pJC} 175 \mathrm{e}^{11}$. Samples were checked periodically for the presence of recombinant phage bearing pIII by plaquing on S2060 without pJC175e.

Statistics and reproducibility. All assays and LC-MS quantification presented in this work were repeated independently at least three times and provided reproducible results.

Reporting summary. Further information on research design is available in the Nature Research Reporting Summary linked to this article.

\section{Data availability}

Data supporting the findings of this study are available within the paper and its Supplementary Information files. A reporting summary for this article is available as a Supplementary Information file. The datasets generated and analyzed during the current study (including LC-MS data files of Fig. 2g and Supplementary Figs. 10, 11 and 12) are available from the corresponding author upon request. Source data are provided as a Source Data file.

Received: 13 July 2020; Accepted: 29 July 2020; Published online: 21 August 2020

\section{References}

1. Fischbach, M. A., Walsh, C. T. \& Clardy, J. The evolution of gene collectives: how natural selection drives chemical innovation. Proc. Natl Acad. Sci. USA 105, 4601-4608 (2008).

2. Baquero, F., Bouanchaud, D., Martinez-Perez, M. C. \& Fernandez, C. Microcin plasmids: a group of extrachromosomal elements coding for low-molecularweight antibiotics in Escherichia coli. J. Bacteriol. 135, 342-347 (1978).

3. Vior, N. M. et al. Discovery and biosynthesis of the antibiotic bicyclomycin in distantly related bacterial classes. Appl Environ. Microbiol 84, e02828-17 (2018).

4. Kominek, J. et al. Eukaryotic acquisition of a bacterial operon. Cell 176, 1356-1366 (2019)

5. Smanski, M. J. et al. Synthetic biology to access and expand nature's chemical diversity. Nat. Rev. Microbiol. 14, 135-149 (2016).

6. Ravikumar, A., Arzumanyan, G. A., Obadi, M. K. A., Javanpour, A. A. \& Liu, C. C. Scalable, continuous evolution of genes at mutation rates above genomic error thresholds. Cell 175, 1946-1957 (2018).

7. Crook, N. et al. In vivo continuous evolution of genes and pathways in yeast. Nat. Commun. 7, 13051 (2016).

8. Halperin, S. O. et al. CRISPR-guided DNA polymerases enable diversification of all nucleotides in a tunable window. Nature 560, 248-252 (2018)

9. Esvelt, K. M., Carlson, J. C. \& Liu, D. R. A system for the continuous directed evolution of biomolecules. Nature 472, 499-503 (2011).

10. Bryson, D. I. et al. Continuous directed evolution of aminoacyl-tRNA synthetases. Nat. Chem. Biol. 13, 1253-1260 (2017).

11. Carlson, J. C., Badran, A. H., Guggiana-Nilo, D. A. \& Liu, D. R. Negative selection and stringency modulation in phage-assisted continuous evolution. Nat. Chem. Biol. 10, 216-222 (2014).

12. Badran, A. H. et al. Continuous evolution of Bacillus thuringiensis toxins overcomes insect resistance. Nature 533, 58-63 (2016).

13. Patteson, J. B., Cai, W., Johnson, R. A., Santa Maria, K. C. \& Li, B. Identification of the biosynthetic pathway for the antibiotic bicyclomycin. Biochemistry 57, 61-65 (2018).

14. Meng, $\mathrm{S}$. et al. A six-oxidase cascade for tandem $\mathrm{C}-\mathrm{H}$ bond activation revealed by reconstitution of bicyclomycin biosynthesis. Angew. Chem. Int Ed. Engl. 57, 719-723 (2018).

15. Skordalakes, E., Brogan, A. P., Park, B. S., Kohn, H. \& Berger, J. M. Structural mechanism of inhibition of the rho transcription termination factor by the antibiotic bicyclomycin. Structure 13, 99-109 (2005).

16. Miyoshi, T., Miyari, N., Aoki, H., Kosaka, M. \& Sakai, H. Bicyclomycin, a new antibiotic. I. Taxonomy, isolation and characterization. J. Antibiot. 25, 569-575 (1972).

17. Skinnider, M. A., Johnston, C. W., Merwin, N. J., Dejong, C. A. \& Magarvey, N. A. Global analysis of prokaryotic tRNA-derived cyclodipeptide biosynthesis. BMC Genomics 19, 45 (2018).

18. Bonnefond, L. et al. Structural basis for nonribosomal peptide synthesis by an aminoacyl-tRNA synthetase paralog. Proc. Natl Acad. Sci. USA. 108, 3912-3917 (2011).

19. Wang, T., Badran, A. H., Huang, T. P. \& Liu, D. R. Continuous directed evolution of proteins with improved soluble expression. Nat. Chem. Biol. 14, 972-980 (2018). 
20. Fischbach, M. A., Lai, J. R., Roche, E. D., Walsh, C. T. \& Liu, D. R. Directed evolution can rapidly improve the activity of chimeric assembly-line enzymes. Proc. Natl Acad. Sci. USA. 104, 11951-11956 (2007).

21. Johnston, C. W. et al. Assembly and clustering of natural antibiotics guides target identification. Nat. Chem. Biol. 12, 233-239 (2016).

22. Cuthbertson, L., Ahn, S. K. \& Nodwell, J. R. Deglycosylation as a mechanism of inducible antibiotic resistance revealed using a global relational tree for onecomponent regulators. Chem. Biol. 20, 232-240 (2013).

23. Phelan, R. M., DiPardo, B. J. \& Townsend, C. A. A high-throughput screen for the engineered production of $\beta$-lactam antibiotics. ACS Chem. Biol. 7, 835-840 (2012).

24. Hubbard, B. P. et al. Continuous directed evolution of DNA-binding proteins to improve TALEN specificity. Nat. Methods 12, 939-942 (2015).

25. Thuronyi, B. W. et al. Continuous evolution of base editors with expanded target compatibility and improved activity. Nat. Biotechnol. 37, 1070-1079 (2019).

26. Kohanski, M. A., Dwyer, D. J. \& Collins, J. J. How antibiotics kill bacteria: from targets to networks. Nat. Rev. Microbiol. 8, 423-435 (2010).

27. Costello, A. \& Badran, A. H. Synthetic biological circuits within an orthogonal central dogma. Trends Biotechnol. https://doi.org/10.1016/j.tibtech.2020.05.013 (2020).

28. Braffman, N. R. et al. Structural mechanism of transcription inhibition by lasso peptides microcin J25 and capistruin. Proc. Natl Acad. Sci. USA 116, 1273-1278 (2019).

29. Travin, D. Y. et al. Structure of ribosome-bound azole-modified peptide phazolicin rationalizes its species-specific mode of bBacterial translation inhibition. Nat. Commun. 10, 4563 (2019).

30. Du., Y. L., Alkhalaf, L. M. \& Ryan, K. S. In vitro reconstitution of indolmycin biosynthesis reveals the molecular basis of oxazolinone assembly. Proc. Natl Acad. Sci. USA 112, 2717-2722 (2015).

31. Isobe, Y. et al. Manumycin polyketides act as molecular glues between UBR7 and P53. Nat. Chem. Biol. https://doi.org/10.1038/s41589-020-0557-2 (2020).

32. Kling, A. et al. Targeting DnaN for tuberculosis therapy using novel griselimycins. Science 348, 1106-1112 (2015).

33. Pluskal, T., Castillo, S., Villar-Briones, A. \& Oresic, M. MZmine 2: modular framework for processing, visualizing, and analyzing mass spectrometry-based molecular profile data. BMC Bioinforma. 11, 395 (2010).

\section{Acknowledgements}

We thank K. Prather and K. Haslinger for assistance with preliminary LC/MS observations; M.A. Skinnider for input during manuscript editing; C. Lewis and the Whitehead Institute metabolite profiling core facility, as well as J. Pacheco and the Broad Institute Metabolomics Platform for assistance with LC/MS measurements. We also wish to thank A. Truman and N. Vior for providing the Pseudomonas BCM expression plasmid
pJH. This work was supported by the Defense Threat Reduction Agency (grant number HDTRA1-15-1-0051), the Paul G. Allen Frontiers Group, the Banting Postdoctoral Fellowship Program (to C.W.J.; grant number 355511), the National Institutes of Health Director's Early Independence Award (grant number DP5-OD-024590), and the Broad Institute of MIT and Harvard.

\section{Author contributions}

C.W.J. and A.H.B. designed experiments, performed experiments, and analyzed data C.W.J., A.H.B., and J.J.C. contributed to study design and manuscript preparation.

\section{Competing interests}

J.J.C. is scientific co-founder and Scientific Advisory Board chair of EnBiotix, an antibiotic drug discovery company. The other authors have no competing interest.

\section{Additional information}

Supplementary information is available for this paper at https://doi.org/10.1038/s41467020-18018-2.

Correspondence and requests for materials should be addressed to J.J.C.

Peer review information Nature Communications thanks the anonymous reviewers for their contribution to the peer review of this work. Peer reviewer reports are available

Reprints and permission information is available at http://www.nature.com/reprints

Publisher's note Springer Nature remains neutral with regard to jurisdictional claims in published maps and institutional affiliations.

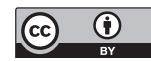

Open Access This article is licensed under a Creative Commons Attribution 4.0 International License, which permits use, sharing, adaptation, distribution and reproduction in any medium or format, as long as you give appropriate credit to the original author(s) and the source, provide a link to the Creative Commons license, and indicate if changes were made. The images or other third party material in this article are included in the article's Creative Commons license, unless indicated otherwise in a credit line to the material. If material is not included in the article's Creative Commons license and your intended use is not permitted by statutory regulation or exceeds the permitted use, you will need to obtain permission directly from the copyright holder. To view a copy of this license, visit http://creativecommons.org/ licenses/by/4.0/

(C) The Author(s) 2020 